\title{
The iCook 4-H Study: An Intervention and Dissemination Test of a Youth/Adult Out-of-School Program
}

\author{
Adrienne A. White, PhD, RDN, FAND ${ }^{1}$; Sarah E. Colby, PhD, RD ${ }^{2}$; \\ Lisa Franzen-Castle, PhD, RD ${ }^{3}$; Kendra K. Kattelmann, PhD, RDN, LN, FAND ${ }^{4}$; \\ Melissa D. Olfert, DrPH, RDN ${ }^{5}$; Tara A. Gould, MS, RDN ${ }^{1}$; Rebecca L. Hagedorn, BS ${ }^{5}$; \\ Douglas R. Mathews, PhD, RD ${ }^{1}$; Jonathan Moyer, $\mathrm{MS}^{6}$; Kimberly Wilson, $\mathrm{MS}^{7}$; \\ Kathryn Yerxa, MS, RD ${ }^{8}$
}

\begin{abstract}
Objective: To describe outcomes from intervention and dissemination of $i$ Cook 4- $H$.

Design: Five-state, community-based participatory research and a randomized, controlled trial followed by a 5-state, nonrandomized dissemination test of the $i$ Cook $4-H$ curriculum with control and treatment groups. Setting: Community and university sites.

Participants: Youths aged 9-10 years and their adult food preparer; 228 dyads in the intervention and 74 dyads in dissemination.

Intervention(s): Theoretical frameworks were Social Cognitive Theory and the experiential 4-H learning model. Six 2-hour, biweekly sessions on cooking, eating, and playing together followed by monthly newsletters and boosters until 24 months, expanded to 8 sessions for dissemination.

Main Outcome Measure(s): Youth body mass index (BMI) z-scores, measured height and weight, and youth/adult program outcome evaluations surveys.

Analysis: Linear mixed models, group, time, and group $\times$ time interaction for BMI z-score and program outcomes changes. Significance levels $=P \leq .05$; interaction term significance $=P \leq .10$.

Results: In intervention, treatment BMI z-scores increased compared with controls based on significant interaction $(P=.04)$. For odds of being overweight or obese at 24 months, there was no significant interaction $(P=.18)$. In dissemination, based on significant interaction, treatment youths increased cooking skills $(P=.03)$ and treatment adults increased cooking together $(P=.08)$ and eating together $(P=.08)$ compared with controls.

Conclusions and Implications: $i$ Cook $4-H$ program outcomes were positive for mealtime activities of cooking and eating together. The program can be successfully implemented by community educators. The increase in BMI z-scores needs further evaluation for youths in cooking programs.

Key Words: community-based participatory research, dyads, $i$ Cook 4- $H$, nutrition intervention, obesity prevention (J Nutr Educ Behav. 2019; 51:S2-S20.)

Accepted November 15, 2018.
\end{abstract}

\footnotetext{
${ }^{1}$ School of Food and Agriculture, University of Maine, Orono, ME

${ }^{2}$ Department of Nutrition, University of Tennessee, Knoxville, TN

${ }^{3}$ Nutrition and Health Sciences Department, University of Nebraska-Lincoln, Lincoln, NE

${ }^{4}$ Department of Health and Nutritional Sciences, South Dakota State University, Brookings, SD

${ }^{5}$ Davis College of Agriculture, Natural Resources, and Design, Division of Animal and Nutritional Sciences, West Virginia University, Morgantown, WV

${ }^{6}$ School of Public Health and Health Sciences, Department of Biostatistics and Epidemiology, University of Massachusetts, Amherst, MA

${ }^{7}$ Department of Health and Nutritional Sciences, South Dakota State University, Extension, Brookings, SD

${ }^{8}$ University of Maine Cooperative Extension, Orono, ME

Conflict of Interest Disclosure: The authors have not stated any conflicts of interest.

The publication of this supplement to the Journal of Nutrition Education and Behavior, including this article, was supported by the National Institute of Food and Agriculture, US Department of Agriculture, under award number 2012-68001-19605. This article underwent the usual peerreview process followed by the journal.

Address for correspondence: Adrienne A. White, PhD, RDN, FAND, School of Food and Agriculture, University of Maine, 5735 Hitchner Hall, Orono, ME 04469-5735; E-mail: awhite@maine.edu

(C) 2019 The Authors. Published by Elsevier, Inc. on behalf of the Society for Nutrition Education and Behavior. This is an open access article under the CC BY license. (http://creativecommons.org/licenses/by/4.0/)

https://doi.org/10.1016/j.jneb.2018.11.012
} 


\section{INTRODUCTION}

iCook 4- $\mathrm{H}$ is an out-of-school program for 9- and 10-year-old youths and their main adult food preparer to cook, eat, and play together for healthful lifestyles. The obesity prevention program was prepared for broad dissemination using a community-based participatory research (CBPR) approach over 6 years of study. A CBPR partnership of researchers, Cooperative Extension faculty including 4-H staff, students, stakeholders, and members of the target population collaborated to increase the likelihood of effective program design, implementation, evaluation, and sustainability. ${ }^{1-3}$ In CBPR, community members are recognized for their knowledge and expertise as they team with academic researchers to accomplish their work. Both the process of working together and the outcome of the project are emphasized in CBPR. ${ }^{4-6}$ For programs to be successfully disseminated, it is critical for researchers to work in partnership with community members. ${ }^{7}$ For the current study, the integration of researchers and Extension personnel offered an existing system for youth programming implementation and sustainability. ${ }^{8,9} \quad 4-\mathrm{H}$ is the largest youth development organization in the US; it reaches almost 6 million youths with a focus on intentional engagement with adult role models and experiential learning on citizenship, science, and healthy living. This learn by doing approach ${ }^{10}$ and the Social Cognitive Theory (SCT) ${ }^{11}$ were the frameworks for building family togetherness and reciprocal role modeling into a program for developing cooking skills, increasing and enhancing family mealtime, and being physically active. The dyad model was used because parents and other caretakers have significant roles in fostering choices youths make and the behavior they model. ${ }^{12}$

It has been almost a decade since the White House Task Force on Childhood Obesity recommended educating children about the importance of nutrition and encouraging families to be active to solve "the problem of childhood obesity within a generation." ${ }^{13}$ Yet, Skinner et $\mathrm{al}^{14}$ found no decline in obesity prevalence in any age group based on the National
Health and Nutrition Examination Survey, 1999-2014. Although healthful behavior developed during youth can lead to positive habits for a lifetime, ${ }^{15-17}$ navigating the complex food environment, lacking kitchen skills and confidence, and feeling challenged by competing demands on time can synergistically build barriers to home cooking and food preparation. ${ }^{18-20}$

In this article, the objective was to describe the 5-state iCook 4-H Study outcomes from the intervention phase (Intervention Study) and dissemination phase (Dissemination Study). Although control and treatment intervention study designs were used in both studies, the Intervention Study was a randomized, controlled trial whereas the Dissemination Study was not randomized. The Dissemination Study was defined as a test of the 8-session iCook $4-H$ curriculum $^{21}$ in the natural or practice environment with minimal researcher intervention and funding. The overall aim of the iCook 4-H Study was to increase cooking competence, family mealtimes, and physical activity of youths to have an impact on the incidence of childhood obesity.

\section{METHODS}

The iCook 4-H Study was conducted in 5 states and was composed of a 2-year Intervention Study and 4-month Dissemination Study. Participants were family dyads of 9- and 10-year-old youths and the adults who were the main food preparers. As depicted in Figure 1, both the intervention and dissemination were pilot-tested in a treatment-only design with 53 dyads in the pilot intervention and 27 dyads in the pilot dissemination before each respective study phase was implemented. In the Intervention Study $(\mathrm{n}=228$ dyads at 0$)$, assessments were conducted at $0,4,12$, and 24 months and included both research measurements (ie, physical measurements, surveys) and program outcome evaluations (ie, curriculum evaluations). The intervention was a curriculum of 6 2-hour, biweekly sessions. In the Dissemination Study, program outcome evaluations were conducted at 0 and 4 months with 74 youths and 76 adults. The intervention was an expanded curriculum of 8 2-hour, biweekly sessions. The CBPR collaborators for the iCook 4-H Study were from the 5 states of Maine, Nebraska, South Dakota, Tennessee, and West Virginia. Study hypotheses are listed in Table 1 . The institutional review board committees for the protection of human subjects at the 5 state universities approved the 6year study. At each study phase, adults provided written consent and youths provided assent for participation. Photo release permission from adults and youths were also provided.

\section{Use of CBPR to Accomplish iCook 4-H Study}

The researchers used CBPR as the structure for the iCook 4-H Study. ${ }^{1-7}$ A systematic approach was undertaken to aid communication and coordination across the 5 states. Drawing on the strengths of the team, and in keeping with the CBPR process, each major study component (eg, recruitment, curriculum development, measurement and evaluation) was managed by subcommittees of investigators, which included Cooperative Extension nutrition specialists; students; experts in family development and exercise science; and community partners, who were primarily county Extension educators and 4-H staff. Training responsibilities were dispersed among all team members with the goals of shared responsibility and building on the strengths of all partners. Research teams of graduate and undergraduate students were recruited with the intent that they would be part of the study for as long as they were in school. The student management team included an overall study administrator with a campus coordinator at each university. Extension researchers recruited county leaders to lead iCook programs. All personnel were part of the CBPR iCook team; most participated in the monthly administrative conference calls and helped to draft, edit, and review study materials (eg, study manual, guides for leaders and participants, recruitment materials, surveys) and outputs (eg, curriculum, abstracts, manuscripts) using cyclical and iterative processes throughout the 6 years. Trainings on research protocols, which included both didactic and practice sessions, were conducted across and 


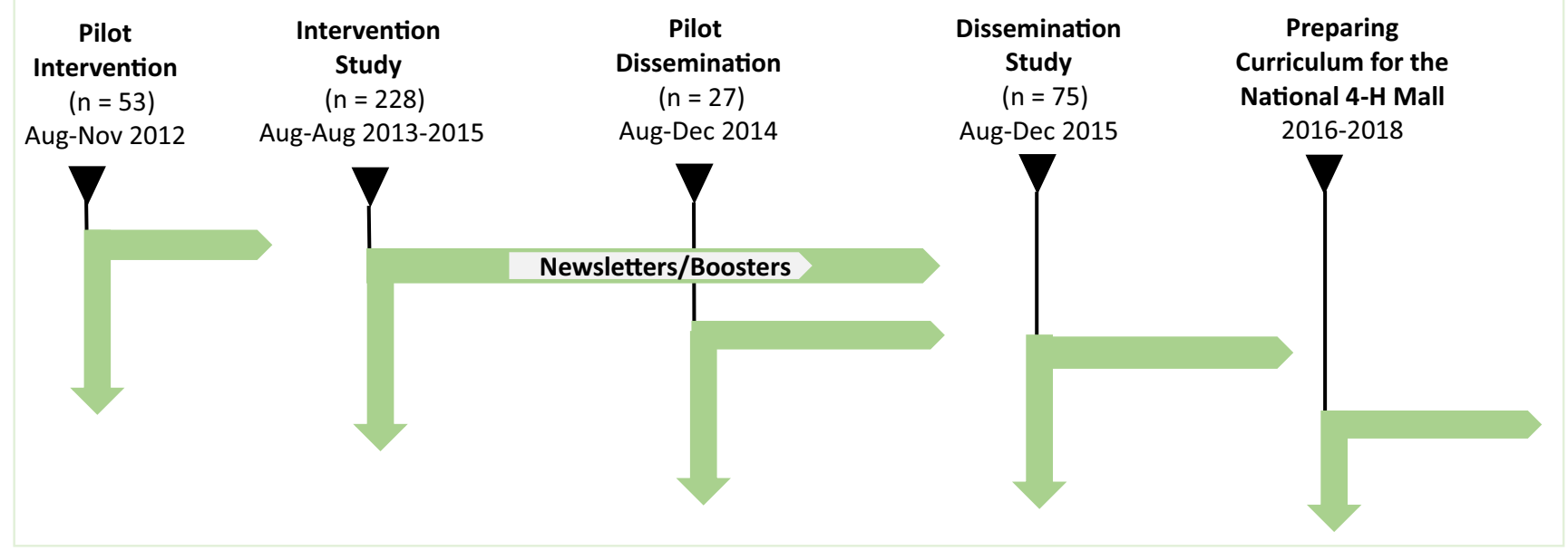

Figure 1. The iCook 4-H Intervention and Dissemination studies were conducted from August, 2012 to December, 2015. Pilot tests solely with treatment groups of each study were conducted. Participants were youths and their adult primary meal preparer. Numbers represent dyads at 0 months of each study. Control participants completed only assessments. Intervention Study treatment participants completed a 6-session iCook 4-H curriculum with follow-up newsletters and booster sessions. Dissemination Study treatment participants completed the expanded 8-session iCook 4-H curriculum. Research and program evaluation assessments were completed for the Intervention Study. Program evaluation assessments were completed for the Dissemination Study. During the final years of the $i C o o k$ 4- $H$ Study, the curriculum was prepared for dissemination through the National 4-H Mall.

within states using the detailed study manual. Trainings, including on the curriculum, were conducted through webinars, videos, in-state face-to-face meetings, and annual 5-state face-to- face meetings. Checklists were used to ensure completion of activities in a timely manner. In the early years of the project, interstate and intrastate advisory committees composed of stakeholders and target dyads provided input into session design. The strengths and resources of community educators, stakeholders, and target population members were

Table 1. Hypotheses Tested for Intervention and Dissemination Studies

\section{Intervention Study: Research Hypotheses}

Hypothesis 1: Over 24 mo, will the change in BMI z-scores for treatment youths be significantly different from that of the control youth?

Hypothesis 2: At 24 mo, for youths who were initially at a healthy BMI, will the odds of being at a healthy BMI for treatment youths be significantly different from the odds for control youths?

Hypothesis 3: At 24 mo, will the odds of staying the same or improving BMI category for treatment youths be significantly different from the odds for the control youths?

Hypothesis 4: Over 24 mo, will the change in selected dietary components for treatment youths be significantly different from that of the control youths?

\section{Intervention Study: Program Evaluation Hypotheses}

Hypothesis 5: Over 4 mo, will the change in the youth and adult program outcome evaluations for the treatment group be significantly different from that of the control group?

Hypothesis 6: Over 24 mo, will the change in the youth and adult program outcome evaluations for the treatment group be different from that of the control group?

\section{Dissemination Study: Program Evaluation Hypothesis}

Hypothesis 7: Over 4 mo, will the change in the youth and adult program outcome evaluations for the treatment group be significantly different from that of the control group? 
realized in the development of an iCook 4-H program that could be disseminated and sustainable at the end of the 6 years.

\section{Intervention Study: 2013-2015}

The Intervention Study was a 2-year, randomized, controlled trial with assessments at $0,4,12$, and 24 months across the 5 states (Figure 1 ). The study was not registered on Clinical Trials.gov. Control and treatment participants received incentives of $\$ 10$ for each youth and adult at each of the 4 assessment periods for a maximum of $\$ 80$. iCook $4-H$ program sites were in locations around each state within driving distance of the universities. County Extension offices, community centers, and university facilities were typical locations where assessments were conducted and interventions were implemented. Research assessments were held on separate days from those of interventions. Screens and small rooms were used to maintain privacy during assessments.

Intervention Study recruitment. The research team recruited a sample of family dyads in late spring and summer before program initiation in September, 2013 (Figure 2). To be eligible, youths had to be aged $\geq 9$ years before the start of the program and not turn 11 years before the end of that year. They were to be free from life-threatening medical illnesses, food allergies, and dietary restrictions and to have access to a computer with the Internet. Body mass index (BMI) category was not a screening criterion. Participating adults had to be the main meal preparer for the youth, with no physical restrictions for movement. Recruiting was targeted for ethnic diversity mostly among rural, low-income communities to meet the need for obesity prevention in those populations.

Based on BMI changes seen in short-term behavioral interventions for youths, ${ }^{22-25}$ power calculations were based on a mean difference in BMI change of $-0.85 \mathrm{~kg} / \mathrm{m}^{2}$ with a common SD of \pm 2.5 , with the suggestion of an effect size of $0.34 \mathrm{~kg} / \mathrm{m}^{2}$. Assuming independent observations, a sample size was calculated to detect this effect size assuming independent observations with a significance level of .05 and power of 0.8 . This resulted in a sample size of 274. Calculations were conducted using SPSS software (version 21.0, SPSS, Inc, Armonk, NY, 2012). An initial sample of 500 youths was based on an attrition of 20\% expected at 4 months and 30\% at 12 and 24 months, based on

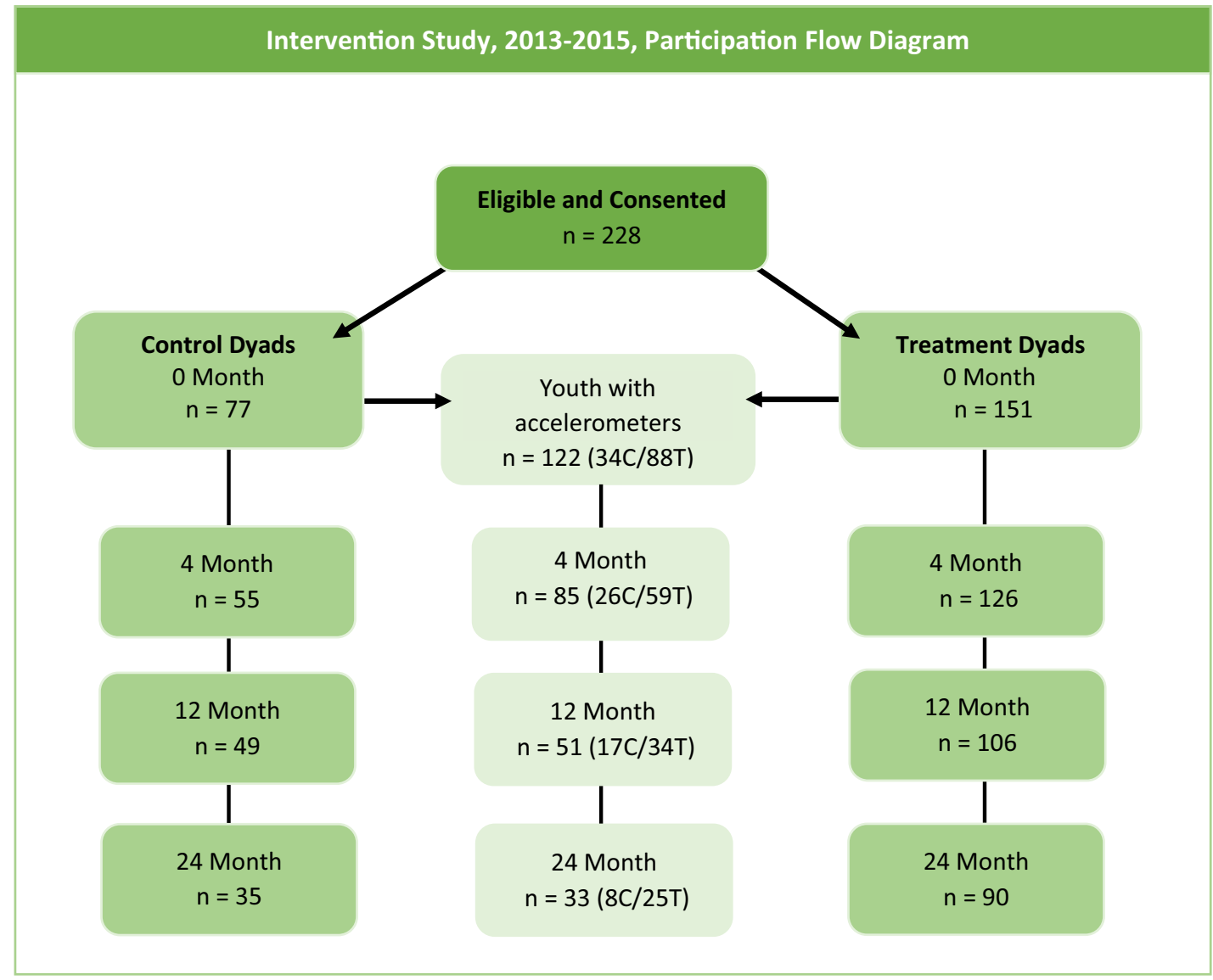

Figure 2. C indicates control; $\mathrm{T}$, treatment. Flow of youth-adult dyad participants through the 2-year iCook 4-H Study. Assessments were conducted at months 0,4,12, and 24. Numbers in the control and treatment dyads flow are based on youths with body mass index data at each month assessment. For the flow of youths with accelerometers, numbers are based on youths who wore accelerometers for 7 days at each assessment period and had reportable data. Only youths who wore accelerometers at 0 months were provided with accelerometers at other periods. 
findings in a previous study with lowincome young adults. ${ }^{26}$ Clustering owing to state was not considered in the initial power analysis. To assess the feasibility of ignoring state-based clustering, the researchers fit a mixed model using observed BMI data over all 4 time points to estimate the variance of a random intercept for state. This model was fit using functionality in the Ime $4^{27}$ package of $\mathrm{R}$ (version 3.4, $\mathrm{R}$ Core Team, Vienna, Austria, 2017). According to the precision offered by the software, the state random effect variance was estimated to be 0 . Based on likelihood ratio test results, there was no significant improvement gained by adding a random effect caused by state to a mixed model without state $(\leq 1)$. Taken together, these results suggested that state-based clustering effects could be ignored.

Recruitment occurred at youth-oriented organizations and clubs, schools and home schools, town halls, churches, pediatrician offices, grocery stores, 4- $\mathrm{H}$ and other Extension e-mail listservs, demonstrations at fairs and day camps, and news releases and other media outlets. Model flyers, media scripts, and letters to community organizations were used across states. Recruited adults received phone calls from researchers to confirm study eligibility, review the consent form, and set appointment times for assessments. To assign dyads to control or treatment groups at each state, a random numbers table ${ }^{28}$ was generated to determine whether a dyad was in the control or treatment group. With about a month of the time left of recruitment, the researchers determined that states were going to fall short of their intended 100 dyads, so in consultation with the statistician, the team decided to use a 1:2 control to treatment randomization to have more treatment than control study participants in the Intervention Study.

Intervention Study research outcome assessments. Research assessments for youths and adults are presented in Table 1. At each assessment period, physical measurements were taken in private settings and secure online surveys were completed using computers or laptops provided by the researchers or participants' personal tablets. Questionnaires were uploaded into an online software program (Qualtrics. com, Qualtrics, Provo, UT, 2013) hosted on a secure server. State campus coordinators were trained and verified on standard anthropometric measurement techniques by the project coordinator. Within each state, student researchers were trained on all protocols and approved to conduct physical asssessments when they met an interobserver reliability of $>0.80 \%$. Physical assessment measures were collected on hard copy forms designed for data collection and verified at each of the 4 assessment periods. Data were entered into an online database, verified by a second researcher by double checking all online entries against the original hard copy data, and submitted for centralized data analyses. The researchers and members of the target population pretested the total assessment survey before administration.

Youths and adults were assessed at $0,4,12$, and 24 months (Figure 2). Youth assessments were anthropometrics, ${ }^{14,29,30}$ Tanner stage for maturation, ${ }^{31-33}$ blood pressure, ${ }^{34-36}$ and survey measurements for dietary intake, ${ }^{37}$ physical activity, ${ }^{38}$ and quality of life. ${ }^{39}$ Adult assessments were multiple surveys (Table 2). ${ }^{40-48}$ Assessments took about 45 minutes to complete. In addition, 155 youths (68\% of the sample) were fitted with accelerometers (GT3X+, ActiGraph, LLC, Pensacola, FL) at 0 months; if these same youths continued in the study, they wore the monitors for 7 consecutive days at each time point. ${ }^{49}$

Intervention Study procedures. The treatment group participated in a curriculum $^{21}$ that was composed of 62 hour, biweekly sessions on cooking, eating, and playing together, called iCook 4- $H$. Dyads received $\$ 10$ (for a maximum of $\$ 60$ ) at each of the 6 sessions they attended, to support intervention-specific costs (eg, travel or food purchases); youths were also given video cameras to record athome cooking, eating, and playing activities (unpublished data, S.E. Colby, PhD, RD, 2018). Each of the 6 sessions included time for youth -adult dyads to cook, eat, be physically active, model mealtime communication, and set goals for healthful lifestyles. MyPlate ${ }^{50}$ was the focus for selecting recipes and meal planning; components of existing 4- $\mathrm{H}$ curricula (Fast Foods! $!^{51}$ and Youth in Motion ${ }^{52}$ ) were modified for the intervention. The CBPR approach was used to gather feedback about the curriculum from stakeholders, session leaders, and the target population. Youths were asked to post videos a minimum of twice per week between sessions on a password-protected website. The website was developed and maintained specifically for the study in a social media-style format for participants to share videos, photos, and conversation about families cooking, eating, and playing together. The $\mathrm{SCT}^{11}$ was operationalized by providing observational learning, reciprocal role modeling between adults and youths, and self-efficacy development and by implementing the $4-\mathrm{H}$ youth development approach of working in partnership with adults in experiential learning. ${ }^{5}$

After the 12-week face-to-face sessions, booster sessions, mailed monthly newsletters, and website challenges were used to continue engagement with the treatment group for the remainder of the 2-year study. Within each state, 3 booster sessions were held about every 6 months with state-specific activities to encourage the theme of cooking, eating, and playing together. Weekly food and monthly physical activity challenges were posted on the website and drawings were held for monetary rewards for meeting the challenges of such things as eating low-fat dairy foods at least $1 \mathrm{meal} / \mathrm{d}$ over a week's time and performing increasing numbers of jumping jacks over a month. The newsletters contained health-related content reflecting the website material.

Intervention Study program outcome evaluation. Measures of program outcome evaluation were developed de novo and with existing resources $^{54,55}$ for the iCook $4-H$ program. The researchers used a 3-pronged approach $^{56}$ for program evaluation, which included program outcome evaluation, ${ }^{57}$ process evaluation, and fidelity of implementation. Process evaluation and fidelity of implementation were addressed in another 
Table 2. Assessment Measures for Youths and Adults Participating in iCook 4-H Study

Measurement

Demographics

Height/weight

Waist circumference 30

Sexual maturation ${ }^{31-33}$

Blood pressure ${ }^{34-36}$

Dietary intake $37,38,40,41$

Physical activity and sedentary time ${ }^{38}$

Eating habits ${ }^{42}$

Family mealtime ${ }^{43}$

Health-related quality of life 39,45

\section{Instrument/ltems}

Youth: state, birth year, grade, sex Adult: state, age, youth's birth date, number of children, marital status, education level, race, youth's race, number in household

SECA 874 digital scale/HealthOMeter $752 \mathrm{KL}$ portable health scale (McCook, IL); SECA 213, SECA Hamburg, Germany/Charder HM200P Portable Stadiometer (Issaquah, WA)

Gulick tape measure (Creative Health Products, Knoxville, TN)

Tanner stage line drawings

Omron digital blood pressure cuff (Warminster, PA)

Youth: Block food screeners for ages 2-17 years, 2007

Adult: NCl Food Scan for Fat; Fruit and Vegetable Screener (Bethesda, MD)

1. Block physical activity screener ages 8-17 years

2. Accelerometers, Actigraph GT3X (Pensacola, FL)

Project Eating and Activity in Teens

Project Eating and Activity in Teens

Pediatric Quality of Life Inventory (PedsQL), version 4.0,

Centers for Disease Control and Prevention

\section{Description}

Youth Adult

Self-reported data obtained from online $\quad X \quad X$ survey

Youth measured, adult self- report Youth: Researcher-trained assessors, using standardized protocols with interrater reliability $\geq 80 \%$ agreement, in-state comparisons

Adult: Self-reported on online survey

Measured on horizontal plane at level of iliac crest and hips at maximum extension of buttocks to nearest $0.1 \mathrm{~cm}$.

Interrater reliability $\geq 0.80 \%$ agreement, in-state comparisons

Self-identified using line drawings of anatomy; parent assisted as requested by youth

Researchers measured using standardized protocols

Youth: Average daily intake of food groups/nutrients eaten in past wk; outcomes measured in cup-equivalents; self-administered, adult assisted as needed

Adult: Food frequency for eating habits over past 12 mo to assess fat intake, fruit and vegetable intake over past mo

1. Frequency and duration of activities in past $7 \mathrm{~d}$; 9 items (leisure, school activities, chores); time per day spent watching television, playing video games, and using Internet; about 5 min to complete by youth with adult assistance, as needed

2. GT3X+ model worn on belt around waist for $7 \mathrm{~d}$; $54 \%$ assigned at 0 months

10 items for when, why, and what is eaten

3 3-item subscales for priority, atmosphere, and structure of meals

23 items for physical, emotional, social, and school or work functioning; self-reported

Assess healthy days (4 items), healthy days symptoms ( 5 items), and activity limitation (5 items) 
Table 2. (Continued)

\section{Measurement}

Kitchen proficiency ${ }^{46}$

Child feeding ${ }^{47}$

Family cohesion $^{44}$
Food security $^{48}$

Program outcome

evaluation $^{56}$

Youth instrument:

Cooking skills

Openness to new foods

Culinary self- efficacy

Adult instrument:

Cooking together

Eating together

Physical activity

\section{Instrument/ltems}

Expanded Food and Nutrition Education Program Behavior Checklist

Birch Questionnaire

Family Adaptability and Cohesion Evaluation Scale IV

Short Form of the US Department of Agriculture Household Food Security Survey Module

De novo or based on existing resources instruments for curriculum ${ }^{54,55}$

\section{Description}

Assessed food resource management, safety, and behavior (11 items)

Assessed restriction (8 items), pressure (4 items), and monitoring ( 3 items) of parent for child to eat

Assessed communication (10 items) and satisfaction (10 items)

Assessed household food insecurity (6 items)

Youths: 23 items, 3 subscales, 7 addi- $\quad X \quad X$ tional items related to curriculum content

Adult: 11 items, 3 subscales
Youth Adult

X

X

$x$

X

$x^{\prime}$
$x$ article about the curriculum. ${ }^{21}$ To test for fidelity, $25 \%$ of the sessions were planned for assessment (40 classes $\times 6$ sessions per class $=$ $240 \times 0.25=60$ sessions). This percentage was based on both consultation with evaluation experts and the number that seemed feasible. To accomplish 60 total evaluations, 10 sessions/state were to be evaluated and a grid was developed so that all sessions would be tested in a scheduled design across the 5 states.

The program outcome evaluation included pre-post instruments for both youths and adults to measure cooking, eating, and playing together. They were designed with Likert-style questions and administered online with the research surveys. Whereas the initial items were used throughout the 2-year intervention, preliminary psychometric testing occurred during the intervention phase $^{56}$ and continued throughout the 6-year study, culminating in instruments that were tested for dimensionality, reliability, and validity in preparation for accompanying the iCook $4-H$ curriculum when distributed nationally. ${ }^{57}$ The finalized instruments were used to obtain program evaluation outcomes. The youth instrument was 23 items (score range, 23-115) and the adult instrument was 11 items (score range, 11 -55). The youth instrument was composed of cooking skills (7 items; $\alpha=.77$ ), openness to new foods (3 items; $\alpha=.79$ ), and culinary self-efficacy (6 items; $\alpha=.83$ ), with additional items retained because of their importance to the curriculum, including 2 items on eating together as a family, 3 on physical activity, and 2 on goal setting. The adult tool was composed of cooking together (5 items; $\alpha=.62$ ), eating together (3 items; $\alpha=.79$ ), and physical activity (3 items; $\alpha=.59$ ). Although Cronbach $\alpha$ 's were lower for 2 of the scales than the desired .7 for good internal consistency, ${ }^{58,59}$ there was evidence for the other psychometric characteristics of adequate dimensionality and validity, as detailed in the article by Mathews et al. ${ }^{57}$

\section{Test of Dissemination: Fall, 2015}

Dissemination Study design, recruitment, and protocols. The 5-state Dissemination Study was conducted in fall, 2015 with the goal of testing the iCook 4 - $H$ program in practice settings, as it would occur without research funding and researcher involvement. It was a nonrandomized design for control and treatment groups with online pre-post assessments and minimal researcher involvement. In preparation for the dissemination, 2 sessions were added to the iCook $4-H$ curriculum to allow for increased time for practicing culinary skills, performing physical activities, and using the website, which was necessary content expressed by previous program leaders, ${ }^{21,49}$ as well as conducting pre-post assessments, which had been included during separate research assessment days for the Intervention Study. As depicted in Figure 1, the Dissemination Study was composed of the iCook 4- $\mathrm{H}$ 8-session curriculum.

Extension partners and researchers recruited 21 community educators to be program leaders for the iCook 4 - $H$ program and 12 formally completed the sessions, which yielded data for 12 programs. The 9 educators who did not complete the program, because of intervening priorities or low program initial enrollment, were from 4 of the 5 states. The iCook 4 - $H$ staff of researchers and partners provided resources, study protocols, and training for program 
leaders through a cross-state webinar and in-state training meetings, during which resources were distributed. Training materials were also available through an online training platform course hosted on eXtension Campus (USDA National Institute of Food and Agriciulture, New Technologies for Ag Extension, Washington, DC; 2018). ${ }^{60}$ Study protocols, resources, and training were consistent with those of the Intervention Study so that testing of the dissemination could occur by program leaders implementing the curriculum without direct involvement by researchers. After training, program leaders independently recruited participants, secured consent forms, conducted program outcome evaluations, and implemented the intervention curriculum. Leaders followed protocols for participant criteria (eg, youth age, lack of dietary restrictions) and recruited from existing $4-\mathrm{H}$ clubs and local elementary schools. While treatment dyads completed the online pre-post assessments at the iCook 4-H sessions, control youths completed assessments during another 4-H program in which they were participating or at their home, with the adult participant.

Sessions were held in a variety of locations identified by researchers and Extension staff and included university campuses, Extension county offices, schools, and community centers with adequate cooking and technology accommodations. Stipends of $\$ 10$ were given to control and treatment youths and adults at each of the 2 pre-post assessment periods for a total of $\$ 40$ maximum/dyad. Video cameras were offered to treatment youths; however, some youths preferred to use their own technology (i.e., smartphones or tablets).

Dissemination program outcome evaluation. The 3-pronged approach ${ }^{56}$ to program evaluation was used during the dissemination phase, the program outcome evaluation, ${ }^{57}$ process evaluation, and fidelity of implementation. Program outcome evaluations, which were conducted on a secure online platform (Qualtrics, 2013) at 0 and 4 months, were the 23-item youth and 11-item adult instruments developed and designed to be completed in 10
-12 minutes. Program leaders identified observers to complete fidelity of implementation evaluations independently. Existing forms were modified from 6 to 8 sessions and implemented in $25 \%$ of sessions, according to the Intervention Study protocols. ${ }^{56}$ Structural (eg, objectives, timing) and instructional components (eg, dyad engagement; leader effectiveness)

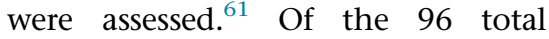
planned sessions (8 sessions/program $\times 12$ programs), 24 were identified for fidelity testing. Online process evaluation, completed at the end of the sessions by youths, adults, and leaders, were used for program monitoring; they were provided to leaders upon request and were used for curriculum revisions. ${ }^{21}$

\section{Statistical Analyses}

Summary statistics were obtained at all points for the Intervention and Dissemination studies. The researchers assessed differences between control and treatment groups at 0 months using 2-sample $t$ tests for continuous variables and chi-square tests of independence for categorical data. Nonparametric versions of these tests (Wilcoxon rank sum test and Fisher exact test, respectively) were used when there were violations in assumptions.

Linear mixed models and logistic mixed models were used to estimate treatment effects over time using $\mathrm{R}$ (version 3.4, R Core Team, Vienna, Austria, 2017) and utilities in the Ime $4^{27}$ and ImerTest $^{62}$ packages. Fixed effects included group, time, and the group $\times$ time interaction. Random effects included random intercepts and time slopes for individual youths. Ordinary logistics regression was used to fit models with nonlongitudinal dichotomous outcomes. Model fit was assessed using residuals plots appropriate to the nature of outcome variables. ${ }^{63}$

The researchers employed likelihood ratio tests to determine whether adding the group $\times$ time interaction offered an improvement over the reduced model with no interaction. $P=.10$ was used to assess the significance of the interaction to improve power, as described by Selvin. ${ }^{64}$ Post hoc contrasts of within-group changes for significant interactions were done using tools in the multcomp ${ }^{65} \mathrm{R}$ package (R Core Team). Effect sizes for linear mixed-model interactions were calculated using a mixed-model variation of Cohen's $\mathrm{d}^{66,67}$ given by Westfall et $\mathrm{al}^{68}$ and interpreted in a similar manner (eg, 0.2 is a small effect). Odds ratios were reported as the effect size for logistic models.

Two strategies were used to account for missing data in the Intervention Study. The first consisted of adjusting the group $\times$ time interaction model with BMI z-score as the outcome by fitting several combinations of demographic and socioeconomic covariates, assuming a missing at random mechanism for missing values. ${ }^{69}$ To compare these non-nested models, an information criterion approach was used for model selection, ${ }^{70}$ using marginal Akaike information criteria and marginal Bayesian information criteria (BIC), a more conservative approach. ${ }^{71}$ Covariates, adult marital status, adult use of government assistance programs, and youth race were determined by the lowest marginal Akaike information criteria and low marginal BIC. Although only use of government programs and youth race were present in the best model using marginal BIC, owing to the relatively strong correlation between marital status at 0 months and a complete case indicator (Cramér's $\mathrm{V}=0.32$ ), it was retained as a covariate. These strategies were not used for the dissemination data owing to the modified demographic information asked of participants in the study. The multivariate imputation by chained equations algorithm ${ }^{69}$ was used to impute missing data for BMI z-score values. Variables strongly correlated with incomplete data status (eg, adult marital status, adult use of government programs, youth race, state, parental education, adult BMI, and youth BMI z-score) were used as imputation variables to impute 100 data sets. The covariate-adjusted interaction model was fit to each of the imputed data sets, the results were pooled together, and the mean interaction effect estimate was calculated.

The researchers performed several secondary analyses to determine the impact of other factors besides group. A dose variable was created based on 
the number of sessions the youths attended. A full dose was determined to be a youth who attended 5 or 6 sessions, whereas a not-full dose was attending $<4$ sessions. To assess the effect of dose, in the covariateadjusted models, group was replaced with dose. In addition, bivariate summaries of the outcome for stayed healthy and maintainers, respectively were calculated using values at 0 months of the various characteristics.

\section{RESULTS}

\section{Intervention Study Youth and} Adult Descriptions at 0 Months

At 0 months, 228 dyads (control, $\mathrm{n}=77$; treatment, $\mathrm{n}=151$ ) completed assessments. Overall, youths (mean age, 9.35 years; SD, 0.67 years) were $55 \%$ female and $68 \%$ were white. Diversity was represented by $14 \%$ Hispanic and $12 \%$ black youths. Adult demographic characteristics are listed in Table 3. Adult males, who made up $10 \%$ of the sample, were older than females $(P=.05)$. Using the youth BMI z-score at all 4 assessment points to distinguish study completers from non-completers, adults were more likely not to complete the study if they were nonwhite $(P=.02)$, were not married $(P<.001)$, were overweight $(P=.01)$, were less educated $(P<.001)$, and used government assistance programs $(P=.005)$. Dropouts were more likely to be in the control vs treatment group $(P=.05)$. Attrition at 4 months was $20 \%$ as expected, but rather than the 30\% expected at 12 and 24 months, it was $15 \%$ and $19 \%$, respectively.

In Table 4, youth and adult physical assessments at 0 months are shown. When significant differences by group (control and treatment) and sex (male and female) were noted, findings are shown. Based on measured heights and weights for youth, mean BMI z-score (0.62; SD, 1.14) was in the upper range of healthy, falling at the 75 th percentile growth curve, ${ }^{29}$ whereas $21 \%$ of youths were in the

Table 3. Demographic Self-Reported Characteristics of Women $(n=188)$ and Men $(n=21)$ in Intervention Study

$\begin{array}{lcc}\text { Characteristic } & \text { Women, } \mathbf{n}(\%) & \text { Men, } \mathbf{n}(\%) \\ \text { Mean age, y (mean [SD]) } & 43(11)^{\mathrm{b}} \\ \text { Race } & 38(5) & 17(81) \\ \text { White } & 138(75) & 0 \\ \text { Hispanic } & 25(14) & 3(14) \\ \text { Black } & 13(7) & 1(0.02) \\ \text { Other } & 7(4) & \\ \text { Marital status } & & 11(52) \\ \text { Married } & 134(72) & 10(48) \\ \text { Not married } & 53(28) & 11(52) \\ \text { Education } & & 10(48) \\ \text { Post high school degree } & 108(57) & 9(45) \\ \text { High school degree or less } & 80(43) & \\ \text { Low-income program participation } & 73(40) & 7(34) \\ \text { General health assessment } & & 11(52) \\ \text { Excellent/very good health } & 77(41) & 3(14) \\ \text { Good } & 77(41) & 34(18) \\ \text { Fair/poor health } & \end{array}$

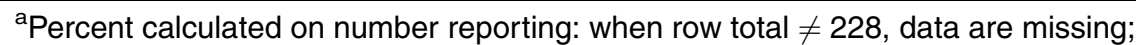
${ }^{\mathrm{b}} \mathrm{P}=.05$, men were significantly older based on a 2-sample $t$ test; ${ }^{\mathrm{C}}$ Responses included Asian, Native American, and other; ${ }^{\mathrm{d}}$ Program options included Aid to Dependent Children, Temporary Assistance to Needy Families, Expanded Food and Nutrition Education Program, Free and Reduced-Price School Meals, Medicaid, Welfare-to-Work, Special Supplemental Nutrition Program for Women, Infants, and Children, and Supplemental Security Income; ${ }^{\text {e}}$ Centers for Disease Control and Prevention Quality of Life. ${ }^{45}$

obese category. In female youths, a group difference was noted for the Tanner stage of maturation for breast development (Tanner female upper); treatment females were more likely to be in prepuberty than were controls $(P=.03){ }^{31,32}$ Most youths $(88 \%)$ had normal blood pressure. Based on selfreport, adult mean $\mathrm{BMI}$ was right at the obese level $\left(29.72 \mathrm{~kg} / \mathrm{m}^{2}\right.$; SD, 7.51 $\mathrm{kg} / \mathrm{m}^{2}$ ) and $41 \%$ were in the obese category. Most adults had normal or prehypertensive blood pressure levels; sex differences $(P<.001)$ were noted. ${ }^{72}$

Survey characteristics of youths and adults at 0 months are presented in Table 5. Youth characteristics for quality of life, eating habits, physical activity, and evaluation of the iCook 4- $H$ program and adult characteristics for quality of life, child feeding, eating habits, and program outcome evaluation are shown. Sex differences were observed, especially for dietary intake in youths. Females reported lower intakes $(P \leq .02)$ for kilocalories, macronutrients, and some dietary components, but were similar to males for dietary fiber, fruits, vegetables, dairy, legumes, and added sugar. Few group differences were noted; however, treatment vs control youth had higher cooking skills $(P=.01)$. In addition, adult mean fruit and vegetable intake was 3.3 cup-equivalents/d (SD, 2.7) and there were no differences by group $(P=.72)$ or $\operatorname{sex}(P=.53)$.

\section{Intervention Study Results of Research Outcomes}

Change in youth BMI Z-scores in Intervention Study. Changes in research outcomes for youth during the Intervention Study over 24 months are compared in Table 6. Hypotheses are shown in Table 1. For hypothesis 1, after adjusting for confounders, youth race (ie, white or nonwhite), adults' marital status (ie, married or not married), and adults' use of government assistance programs at 0 months, there was a significant interaction between group and time $(P=.02)$. The control group change in BMI z-scores of $-0.055 / y$ was nonsignificant $(P=.15)$ but the observed change in the treatment group of $0.051 / y$ was significant 
Table 4. Youth and Adult ( $n=228$ Dyads) Physical Assessments at 0 Months of Intervention Study

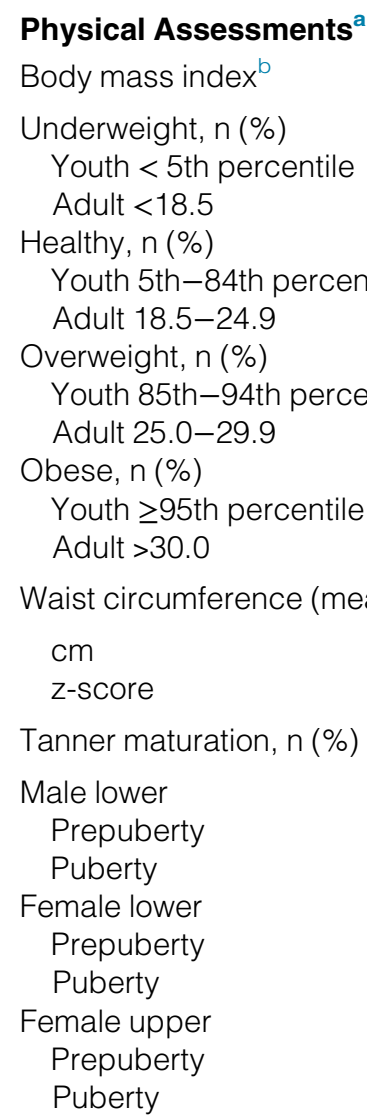

Tanner matura
Male lower
Prepuberty
Puberty
Female lower
Prepuberty
Puberty
Female upper
Prepuberty
Puberty

Youths, z-score

$0.62(1.14)$

$$
8(3.5)
$$

$133(58.3)$

$40(17.5)$

$47(20.6)$

$68.8(12.19)$

$0.095(1.05)$

$$
\begin{array}{cr}
34(59) \\
24(41) \\
44(66) \\
23(34) \\
\multicolumn{2}{c}{34(44)} \\
\text { Control } \quad \text { Treatment } \\
6(18) & 28(82) \\
44(56) & \\
\text { Control } & \text { Treatment } \\
20(45) & 24(55)
\end{array}
$$

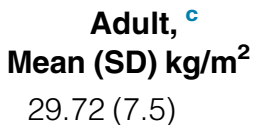

$$
2(1.0)
$$$$
62(30.5)
$$

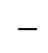

$-$

\begin{tabular}{|c|c|}
\hline \multicolumn{2}{|c|}{$136(66)$} \\
\hline Female & Male \\
\hline $132(71)$ & $4(20)$ \\
\hline \multicolumn{2}{|c|}{$70(34)$} \\
\hline Female & Male \\
\hline 54 (29) & $16(80)$ \\
\hline
\end{tabular}

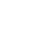

$-$

$-$

$-$

\section{Systolic blood pressure risk category, n (\%)}

Normal

198 (87.6)

$28(12.3)$
${ }^{a}$ Data presented by youths and adult; group and sex differences are detailed when significances were observed. ${ }^{29-33,35,36}$ Youth waist circumference reference data: males, 69.2 (1.09); females, 69.8 (9.7). ${ }^{30}$ Tanner stage of maturation assessment was conducted in 3 of 5 iCook $4-H$ states $^{32,33}$; ${ }^{b}$ Group differences in Tanner stage for female breast development (Tanner upper) $(P=.03)$, based on $\chi^{2}$ test of independence; ${ }^{c}$ Adult sex differences in blood pressure risk category $(P<.001)$, based on $\chi^{2}$ test of independence.

$(P=.05)$. Over time, BMI Z-scores for treatment youth significantly increased, whereas BMI z-scores for controls did not. However, the finding for the analysis of the interaction effect with imputed data (not shown in the table) was not significant; it yielded an estimated interaction effect of 0.01 (90\% confidence interval, -0.02 to 0.03$)$.
When the Tanner ${ }^{31,32}$ upper stage for females was added as a confounder, there was a significant, weak interaction effect $(d=0.01$; $P=.03),{ }^{66-68}$ indicating that control females had significantly decreased BMI z-scores $-0.11 / y(P=.02)$ and no effect was seen for treatment females $(P=.63)$. Based on logistic mixedmodel testing as to whether Tanner upper stage was related to the movement of females into the combined category of overweight and obese (overweight/obese), there was no significant interaction $(P=.14)$, which indicated that treatment females were no more likely than control females to move into the overweight/obese category, and thus risk for becoming overweight or obese was the same for both groups that were assessed for Tanner upper stage.

Results for hypotheses 2 and 3 are presented in text only. For hypothesis 2, using unadjusted analysis, the odds did not differ (odds ratio $[\mathrm{OR}]=0 ; \quad P=.06$ ) for control and treatment youths moving out of the healthy range. Similarly, for hypothesis 3, after adjusting for the confounders, the odds did not differ (OR $=.42 ; P=.21)$ for control and treatment youths staying the same or improving in the BMI category. Overall, the findings were that the odds of youths being overweight or obese at 24 months did not differ by group.

Change in youth food intake in Intervention Study. Over the 24 months of the Intervention Study, dairy and legume intakes were the only changes in food intake for the youths. For hypothesis 4 , based on the linear mixed-model hypothesis testing, there was a significant interaction between group and time $(\mathrm{d}=.02$, very weak; $P=.06){ }^{66-68}$ Controls decreased dairy foods by -0.26 cup-equivalents/y $(P=.001)$, whereas treatment youths maintained intake by changing only -0.08 cupequivalents/y $(P=.19)$ (Table 6). Based on logistic mixed-model hypothesis testing, there was a significant interaction between group and time for legume intake $(P=.10) \quad(\mathrm{OR}=0.96)$. Using the same 0.10 level of significance for post hoc contrasts as for interactions, there was no significant change for a given control youth $(P=.53)$ whereas the odds of a given 
Table 5. Youth $(n=209)$ and Adult $(n=208)$ Survey Characteristics at 0 Months for Intervention Study

\section{Characteristic $^{a}$}

Pediatric Quality of Life Inventory (mean [SD])

Overall

Physical

Emotional

Social

School/work

Psychological

Centers for Disease Control and Prevention,

Quality of Life ${ }^{d}$

Physically unhealthy $\mathrm{d} / \mathrm{mo}$ (mean [SD])

Total unhealthy $\mathrm{d} / \mathrm{mo}$ (mean [SD])

Adult child feeding characteristics (mean [SD]) ${ }^{\mathrm{e}}$

Restrict access to food

Pressure child to eat more food

Monitor what child eats

Daily eating habits, $\mathrm{n}(\%)$

Eat breakfast

Eat lunch

Eat dinner

Eat fast food

Dietary intake, frequency over past $w^{\dagger}$

Total mean kcal

Protein, $g$

Carbohydrate, $\mathrm{g}$

Saturated fat, $g$

Dietary fiber, $g$

Fruit, cup-equivalents

Vegetables, cup-equivalents

Potatoes, cup-equivalents

Dairy, cup-equivalents

Legumes, cup-equivalents

Meat/poultry/fish, oz/equivalents
Youths

$77.9(14)$

$81.7(17)$

$71.2(19)$

$80.0(18)$

$76.8(16)$

$76.0(14)$

Control Treatment

73.7 (14) $77.1(15)$

$-$

$-$

$-$

$-$

$-$

$143(76)$

$160(87)$

$167(92)$

6 (3)

1,331 (914)

Female Male

$1,200(723) \quad 1,492(1,089)$

$$
\begin{array}{lc}
\multicolumn{2}{c}{58(45)} \\
\text { Female } & \text { Male } \\
51(30) & 67(57)
\end{array}
$$

$$
\begin{array}{lc}
\multicolumn{2}{c}{161(99)} \\
\text { Female } & \text { Male } \\
151(92) & 174(106)
\end{array}
$$

$$
\begin{array}{cl}
\multicolumn{2}{c}{19(15)} \\
\text { Female } & \text { Male } \\
17(11) & 22(18)
\end{array}
$$

$$
\begin{aligned}
& 12(8.1) \\
& 1.6(1.1) \\
& 0.8(0.7) \\
& 0.3(0.3)
\end{aligned}
$$

Female Male

$0.27(0.34) \quad 0.31(0.28)$

$1.7(1.1)$

$0.06(0.1)$

\section{Adult}

$79.6(12)$
$76.1(19)$
$73.5(17)$
$88.2(13)$

Female Male

89 (13) $79(14)$

80.0 (15.1)

$\begin{array}{ll}\text { Female } & \text { Male } \\ 80(14) & 73(18) \\ 80.8(12) & \\ \text { Female } & \text { Male } \\ 81(11) & 75(13)\end{array}$

$2.8(5.9)$

$7.0(9.0)$

$29(6)$

11(2)

$11(3)$

105 (54)

121(62)

$163(84)$

0

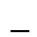

$-$

$-$

$-$

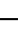

(continued) 


\section{Table 5. (Continued)}

Characteristic $^{\mathrm{a}}$

Whole grains, oz/equivalents

Added sugar, teaspoons

Physical activity, moderate/vigorous, min/d (mean [SD])

Program outcome evaluation, mean (SD)

Youth scales ${ }^{\mathrm{g}}$

Cooking skills, ${ }^{\mathrm{h}} \alpha=.77$

Openness to new food, $\alpha=.79$

Culinary self-efficacy, $\alpha=.83$

Adult scales

Cooking together, $\alpha=.62$

Eating together, $\alpha=.79$

Physical activity, $\alpha=.59$

\section{Youths}

$3.0(0.1)$

Female Male

2.4 (1.9) $3.5(3.9)$

$$
\begin{gathered}
0.7(0.7) \\
\text { Female } \quad \text { Male } \\
0.65(0.72) \quad 0.56(0.72) \\
7.2(6.0) \\
116(97) \\
\\
22.7(7.2) \\
\text { Control } \\
18.7(6) \\
11.6(3.1) \\
22.9(4.6)
\end{gathered}
$$

Adult

$-$
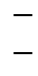

$-$

$16.3(2.7)$

$12.0(2.0)$

$10.5(1.9)$

${ }^{a} \mathrm{n}<228$ dyads indicates missing data; ${ }^{b} P=.05$, youth group differences; treatment vs control reported higher psychological quality of life, ${ }^{39}$ based on Wilcoxon rank sum tests; ${ }^{c}$ Adult gender differences, females vs males reported higher social $(P=.002)$, work $(P=.04)$, and psychological $(P=.02)$ quality of life, ${ }^{39}$ based on Wilcoxon rank sum tests; ${ }^{d}$ Health-related quality of life selected items ${ }^{45}$; ${ }^{e}$ Birch Child Feeding Questionnaire, ${ }^{47}$ Likert scale ranging from $5=$ agree to $1=$ disagree. Possible scores: restriction $=8-40$; pressure to eat $=4-20$; monitoring $=3-15$; ${ }^{f}$ Youth sex differences based on Wilcoxon rank sum tests; females vs males reported lower kcals $(P=.006)$, protein $(P=.008)$, carbohydrates $(P=.04)$, saturated fat $(P=.001)$, potatoes $(P=.02), \mathrm{meat} /$ fish/poultry $(P=.003)$, and whole grains $(P=.02)^{37}$; ${ }^{2} 23$-item, Likert format; score range 23-115; cooking skills: 7 items, $5=$ all the time to $1=$ never, possible score $7-35$; openness to new foods: 3 items, $5=$ very willing to $1=$ very unwilling, possible score 3-15; culinary self-efficacy: 3 items, $5=$ strongly agree to $1=$ strongly agree, possible score 3 -15; additional items retained in instrument owing to curriculum content. Eating together as a family: 2 items, $5=$ all the time to $1=$ never; possible score 2-10; physical activity: 3 items, $5=$ all the time to $1=$ never, possible score $3-15$; goal setting: 2 items, $5=$ all the time to $1=$ never, possible score $2-10^{57}$; houth treatment group had significantly higher cooking skills than controls $(P=.01)$, based on 2 sample $t$ tests; ${ }^{i} 11$-item Likert format, ranging from $5=$ always to $1=$ never, score range, $11-55$; cooking together: 5 items, possible score 5-25; eating together: 3 items, possible score 5-15; physical activity: 3 items, possible score $5-15 .^{57}$

treatment youth consuming at least some legumes decreased by a factor of $0.72 / y(P=.05)$.

Intervention Study program outcome evaluations. Results of hypotheses 5 and 6 testing are presented next. For the Intervention Study, from 0 to 4 months, no between-group differences in program outcome evaluation were noted for control or treatment youths, nor were there significant interactions from 0 to 24 months. However, as shown in Table 7, from 0 to 4 months, there was a significant interaction for adults cooking together; treatment adults significantly increased behavior for cooking together whereas control adults did not $\left(\chi^{2}[1]=4.94 ; P=.03 ; \mathrm{d}=.01\right.$, a weak effect). ${ }^{66-68}$ Based on post hoc contrasts, control adults' change of -0.32 points over 4 months was not significant $(\mathrm{z}=-0.15 ; \quad P=.89)$ whereas treatment adults' change of 0.89 points over 4 months was $(\mathrm{z}=4.26 ; P=.001)$. However, these 4 month gains by treatment adults relative to controls were indistinguishable when analyzed using data across all 4 time points. That is, there was no significant group $\times$ time interaction for adult program outcome evaluation change over 24 months $(P=.46)$.
Fidelity of implementation in Intervention Study. For fidelity testing in the Intervention Study, in the 0- to 4month time frame when the curriculum was implemented, $23 \%$ vs the planned $25 \%$ of 240 sessions were evaluated. Observers $(n=16)$ across the 5 states reported that session objectives were met $96 \%$ of the time. They observed that leaders were very effective or effective $94 \%$ of the time, youths were engaged $90 \%$ of the time, and adults were engaged $80 \%$ of the time. Actual vs planned session length (120 minutes) was a mean of 115 minutes (SD, 10 minutes).

As a measure of dose, of the 126 treatment youths who were 
assessed at 4 months, 63 (50\%) attended all 6 sessions and 108 $(86 \%)$ completed at least 5 of the sessions. Over all 24 months, the rate at which the high-dose youth changed cooking skills was not significantly different from that of low-dose youth $(P=.97)$.

\section{Distinguishing Features of Successful iCook 4-H Youths in Intervention Study}

Distinguishing features emerged for youths in the Intervention Study when the researchers explored characteristics at 0 months for treatment youths $(n=90)$ who completed 24month assessments and could be termed successful in the iCook $4-H$ program based on either staying in the healthy BMI category, stayed healthy, or maintaining BMI z-scores within their initial or better growth curve, maintainers. Youth who stayed heathy had higher protein-dense intakes $(P=.05)$ and their adult food preparer reported a lower mean number of days per month that they were physically unhealthy $(P=.006)$ as well as unhealthy for any reason $(P=.04)$; they also reported more restricting of high-fat and high-sugar foods $(P=.02)$ and less pressuring their youths to eat $(P=.001)$. Maintainers vs non-maintainers had more dairy-dense intake $(P=.03)$ and reported more frequent snacking $(P=.05)$. Non-maintainers had higher BMI z-scores $(P=.007)$ and waist circumference $(P=.04)$; their adult meal preparer reported a higher mean number of unhealthy days per month $(P<.001)$, a sign of lower quality of life, and eating less than they should because they lacked money for food $(P=.02)$, a sign of food insecurity.

\section{Dissemination Study Youths and Adult Description at 0 Months}

For the Dissemination Study, at 0 months, 74 dyads (control $=39$; treatment $=35$ ) completed program outcome evaluations. Overall, youths (mean age, 9.6 years; SD, 0.78 years) were $96 \%$ female and $68 \%$ white. Adults had a mean age of 39 years (SD, 6.6 years) with a mean BMI of
26 (SD, 6); 77\% were married, 70\% had post-high school degrees, and $63 \%$ were employed. Of the $26 \%$ who were enrolled in government assistance programs, more were in the treatment than control group $(P=.004)$.

Program outcome evaluations in Dissemination Study. For hypothesis 7, during the 0- to 4-month Dissemination Study, there were significant between-group changes in program outcome evaluation for both youths and adults (Table 7). For youth cooking skills, there was a significant interaction between group and time $\left(\chi^{2}[1]=4.67 ; P=.03\right)$. Treatment youths increased the mean score on cooking skills 2.5 points more than did control youths; per post hoc contrasts, control youths increased the mean score on cooking skills 1.7 points $(\mathrm{z}=2.28 ; P=.02)$ and treatment youths increased the mean score 6.2 points $(\mathrm{z}=5.09 ; \mathrm{P}<.001)$.

For adults in the Dissemination Study, there were significant interactions between group and time for eating together $\left(\chi^{2}[1]=3.01 ; P=.08\right)$ and cooking together $\left(\chi^{2}[1]=3.01\right.$; $P=.08)$. Over 4 months, treatment adults increased the mean score on eating together by 0.6 points more than did control adults; per post hoc contrasts, the score for controls did not increase significantly $(z=0.12$; $P=.91)$ but that for the treatment group increased 0.6 points $(z=2.55$; $P=.01)$. Similarly, for cooking together, the treatment group increased the mean score 0.7 points more than did controls; per post hoc contrasts, the score for controls did not increase $(\mathrm{z}=.09 ; P=.93)$ but that for treatment increased 0.77 points $(\mathrm{z}=2.53 ; P=.01)$.

Fidelity of implementation in Dissemination Study. For fidelity testing during the Dissemination Study, observers reported that $91 \%$ of all planned objectives were met. They observed that leaders were very effective or effective $97 \%$ of the time, youths were engaged $88 \%$ of the time, and adults were engaged $91 \%$ of the time. Compared with planned (120-minute) session length, the actual length was 119 minutes. 
Table 7. Comparison of Changes in Program Evaluation Outcomes From 0 to 4 Months in Youths and Adults During Intervention and Dissemination Studies

Intervention Study

Assessment mo

Dyads, $n^{a}$

Program evaluation outcome

Adult cooking together

Dissemination Study

Assessment mo

Dyads, $n^{a}$

Program evaluation outcome ${ }^{b}$

Youth cooking skills

Adult eating together

Adult cooking together

\begin{tabular}{cc}
\multicolumn{3}{c}{ Control } \\
0 & \\
77 & 4 \\
& 55
\end{tabular}

Adjusted Mean (SE)

$16.3(0.3) \quad 16.4(0.3)$

Control

$\begin{array}{cc}0 & 4 \\ 63 & 39\end{array}$

Adjusted Mean (SE)

$23.0(0.8) \quad 24.7(0.8)$

$12.0(0.3) \quad 12.0(0.3)$

$12.1(0.4) \quad 12.1(0.4)$

\begin{tabular}{cc}
\multicolumn{2}{c}{ Treatment } \\
0 & 4 \\
151 & 126
\end{tabular}

Adjusted Mean (SE)

$16.3(0.2) \quad 17.2(0.2)$

Treatment

$\begin{array}{cc}0 & 4 \\ 75 & 35\end{array}$

Adjusted Mean (SE)

$22.6(0.9) \quad 26.8(0.9)$

$11.2(0.3) \quad 11.8(0.3)$

$12.7(0.4) \quad 13.5(0.4)$
$\boldsymbol{P}$

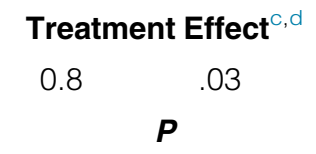

Treatment Effect ${ }^{\mathrm{C}}$

$\begin{array}{ll}2.5 & .03 \\ 0.6 & .08 \\ 0.7 & .08\end{array}$

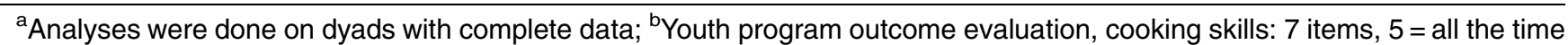
to $1=$ never, score range $7-35$; adult program evaluation, Likert scale, $5=$ always to $1=$ never; cooking together: 5 items, score range 5-25; eating together: 3 items, score range, $3-15$; $^{\text {C}}$ Treatment effect is based on group, time, and group $\times$ time interaction in a linear mixed model. Models included covariates representing participant characteristics of youth race, adult use of government assistance programs, and marital status. $P$ values were obtained with likelihood ratio tests of the no-interaction model vs the interaction model; ${ }^{d}$ Effect size: intervention adult cooking together, $d=.01$, very weak. ${ }^{66-68}$

Note: $P \leq .10$ for significant interactions. ${ }^{64}$

\section{DISCUSSION}

The iCook 4-H Study was a comprehensive project for 9- to 10-year-olds and their primary adult meal preparer to cook, eat, and play together for obesity prevention. The 6-year project included a 2-year longitudinal study and dissemination test of the program in the practice setting to deliver a family-based curriculum to the public domain, including pre-post outcome measures for youths and adults and a tool to evaluate the fidelity of implementation. The project was accomplished through a communitybased participatory research-Extension partnership across 5 states; from the beginning, the aim was to produce a program with the potential for sustainability.

Whereas the motivation behind iCook $4-H$ was obesity prevention, the foundational principle was to promote a healthful lifestyles intervention. Overweight youths were not screened out of this program targeted for rural, low-income families. In fact, $38 \%$ of those recruited were in the overweight or obese BMI categories. The percentage of youths over the 2 years did not change and was consistent with national prevalence. ${ }^{14}$
It was interesting to track height and weight measures for these youths as they aged from 9-10 to 11-12 years over the 2-year Intervention Study. It was surprising to find that although the effect was weak, the BMI z-scores increased for the treatment but not the control group. Kilocalorie intake and physical activity levels, which were measured factors that could be associated with BMI, did not differ by group. This increase in BMI zscores may have been associated with growth variation. There is some support for that because, in girls, of those who were assessed for maturation, more in the treatment than control group were in prepuberty at study initiation. For both sexes in the control and treatment groups, there were no differences in the rate at which they moved toward an unhealthy BMI category. There was no indication that the intervention affected the obesity status of study youths. Findings across studies differed as to the impact of interventions on BMI. Researchers reported trends ${ }^{73}$ or no effect ${ }^{74}$ on BMI changes; others noted BMI reductions when components such as gardening were combined with cooking, as in LA Sprouts, ${ }^{75}$ or when self-management techniques were included with physical activity and nutrition, as in Fit 4 Life. $^{76}$

Dietary changes identified after 24 months for the Intervention Study were minimal. However, the treatment youths maintained dairy intake whereas the controls decreased intake, which could have been because of the strong emphasis in the curriculum ${ }^{21}$ on recipes (eg, for smoothies and parfaits) and meal planning around MyPlate. ${ }^{50}$ Although there was a weak effect on mean intake, which was below the 3 cups recommended for healthful eating, ${ }^{50}$ this was a positive finding for iCook 4- $H$ and also for the treatment youths if they continue to maintain dairy in their diets, because of the potential beneficial effect on obesity risk. ${ }^{77,78}$ In contrast, it was surprising that legume intake was maintained for controls compared with treatment youths because iCook $4-H$ participants cooked with lentils. Intake of both groups was low, but adding legumes such as lentils to dietary choices would contribute to low-fat, vegetarian protein options.

Although iCook 4 - $H$ was an out-ofschool program, aspects differed from 
more traditional programs ${ }^{74,76,79-81}$ since it was not implemented where youths were already gathered for afterschool care and the dyad model has been rarely reported. ${ }^{82}$ In addition, the research protocols were juxtaposed with community setting atmospheres. It is especially important to assess the fidelity of implementation when programs are implemented across multiple states, within community settings, and using the CBPR approach. Results from program observers for fidelity in both intervention and dissemination were positive about objectives being met, timing of sessions being consistent with planned time, and the effectiveness of leaders and engagement of participants, which provided support that programs were implemented as intended.

A qualitative impact evaluation conducted at the end of the 24-month Intervention Study is reported in this issue ${ }^{83}$ to amplify the benefits of the Intervention Study for the treatment group. The researchers used ripple effects mapping $(\mathrm{REM})^{84}$ to assess program effectiveness by identifying participants' perceptions of the program's success for individuals, families, and the broader community. Of the 90 treatment dyads that completed 24-month quantitative measures, 35 (39\%) participated in REM. They identified themes consistent with the $i$ Cook $4-H$ focus on cooking, eating, and playing together for healthful lifestyles, which included increased knowledge of new recipes, preparing one's own meals, looking at food labels, having more fun sharing (when eating) at the table, playing with peers and family leading to better health, and more involvement in the community through volunteerism and being role models. In addition to the REM evaluation, a qualitative assessment of the use of cameras given to youths to make athome videos has been completed (unpublished data, S.E. Colby, PhD, RD, 2018). Mixing, cutting, and measuring were the top 3 cooking techniques that youths demonstrated. The videos were also assessed for the typology of adult interactions with youths; they were identified as being supportive, negative, laid-back, or mixed in their approach while the youths were cooking.

Use of qualitative assessments such as REM and analyses of dyadproduced videos increased the perspective of the iCook $4-H$ program and dyad participants. When analyses were completed to identify characteristics of dyads based on whether youths stayed in the healthy BMI category or maintained their initial BMI category or better, interesting characteristics emerged. Compared with youths in the other BMI categories, over the 24 months, stayed healthy youths had higher protein density intake and adults had signs of higher quality of life. Although they restricted high-fat and high-sugar foods, adults pressured the youths to eat less compared with adult meal preparers for the other 3 BMI categories. Inconsistencies in findings about the relation of parents' reports of feeding practices and children's weight and study designs make direct comparisons difficult. However, in contrast to the current findings, Birch et $\mathrm{al}^{47}$ reported that parents of heavier children used more restriction to food access and less pressure to eat. Faith et $\mathrm{al}^{85}$ concurred that restriction of food was related to higher BMI z-scores, but these scores were lower for children who were pressured to eat. As in the current study, Hennessy et $\mathrm{al}^{86}$ found that restriction was related to lower BMI z-scores, but acknowledged that because of the complex nature of the parent-child relationship, it was important to consider other factors such as parenting styles. In the current study, maintainers had more dairy-dense intakes and snacked more, compared with non-maintainers who had higher BMI z-scores and waist circumference, and whose adults reported signs of lower quality of life and food insecurity. Food insecurity of the Intervention Study dyads was previously studied. ${ }^{87}$

The dyad model was important to the success of iCook $4-H$ to facilitate the collaborative nature of the project and the long-term commitment to the 2-year Intervention Study. When the researchers initially calculated the sample size, the 24-month sample size was expected to be $39 \%$ of the initial sample, but $55 \%$ of the sample completed the study. Nevertheless, although dyads were considered a unique strength of the program, their involvement might have contributed to the primary limitation of the study, which was that the sample size was less than one half the number planned. Although strong recruitment measures were in place and recruiters were diligent, no state team reached its intended sample size, which limited statistical power and generalizability. In the future, offering the iCook 4 - $H$ program where youths are already gathered for after-school care may help recruitment efforts.

Dissemination of the iCook $4-H$ program was tested in an off-shoot study with the Health Science Technology Academy in West Virginia, a program for high school students to participate in researching areas of science, technology, engineering, and mathematics. ${ }^{88}$ Serving as teen teachers with mentoring from their own schoolteachers, high school students led the iCook $4-H$ program with 24 dyads. ${ }^{89}$ There were minimal quantitative changes, but they received positive responses from youths who reported learning about healthy eating, food safety, and the benefits of being physically active. Adults commented on the importance of quality time with their children and the cooking skills their children gained. Based on results, there appears to be flexibility in disseminating the iCook $4-H$ program and perhaps program sustainability fit for individualized community needs.

\section{IMPLICATIONS FOR RESEARCH AND PRACTICE}

The most challenging results to explain might be the lack of significant findings for the program outcome evaluations for the intervention compared with the dissemination. Whereas no changes were seen between control and treatment youths at 4 months or over the 24-month Intervention Study, in the Dissemination Study, the rate of change observed for treatment youths was significantly different from that of the control group for cooking skills. 
Similarly, when comparing adults between 0 and 4 months when the iCook 4-H curriculum was implemented for both the intervention and dissemination, treatment adults in both studies reported changed behavior for cooking together. However, that rate of change was not sustained over the 24-month Intervention Study, which resulted in no difference between the control and treatment groups at 24 mouths. For treatment adults in the dissemination, reported eating together also increased. It is not known what the findings would have been for the Dissemination Study with a 24-month assessment. The positive results seen in the current study may have been due to expanding the number of sessions from 6 to 8 , in addition to the CPBR and iterative processes used to perfect both the curriculum and training of leaders that occurred between the Intervention and Dissemination studies.

The environment established during the dissemination might have had a positive influence because there was a lack of researcher involvement or oversight, and shorter assessments with program evaluations, not a battery of research measurements, were conducted. Finding a program effect for both youths and adults during the Dissemination Study was important because it gave credence that Extension staff and other community leaders could implement iCook $4-H$ in public health settings.

A longer and more intense program may be needed to realize strong and sustainable nutrition and physical activity $^{90}$ lifestyle changes in youths and their adult primary meal preparer to assuage obesity rates. More study is needed to investigate the relation between increased cooking skills and BMI in youth. The iCook team prepared the iCook $4-H$ curriculum for widespread distribution $^{21}$ and a true scaled-up Dissemination Study might be conducted. To facilitate communicating program results to stakeholders, community members, and policy makers, an infographic was developed and tested during the iCook 4-H Study that may be useful to monitor program sustainability. ${ }^{91}$ The Evidence-Based Forecast Capture, Assemble, Sustain, Timelessness framework $^{91}$ provides program information and other data in an electronically generated, community-specific infographic to illustrate program need and community impact.

\section{ACKNOWLEDGMENTS}

Other funding for this material is from US Department of Agriculture Experiment Stations in Maine, Nebraska, South Dakota, and West Virginia. The funding sponsors had no role in the design of the study; in the collection, analyses, or interpretation of data; in the writing of the manuscript, or in the decision to publish the results. Special thank you to the iCook participants, program leaders, and the many graduate and undergraduate students from participating universities who made this research possible.

\section{REFERENCES}

1. Riley P, Jossey R, Nkinsi L, Buhi L. The CARE-CDC health initiative: a model for global participatory research. Am J Public Health. 2001;91:1549-1552.

2. Corburn J. Combining communitybased research and local knowledge to confront asthma and subsistence-fishing hazards in Greenpoint/Williamsburg, Brooklyn, New York. Environ Health Perspect. 2002;110(suppl 2):241-248.

3. Minkler M, Thompson M, Bell J, Rose K. Contributions of community involvement to organizational-level empowerment: the federal Healthy Start experience. Health Educ Behav. 2001;28:607-783.

4. Israel B, Schultz A, Parker E, Becker A. Review of community-based research: assessing partnership approaches to improve public health. Annu Rev Public Health. 1998;19:173-202.

5. Yancey A, Miles O, Jordan A. Organizational characteristics facilitating initiation and institutionalization of physical activity programs in a multi-ethnic, urban community. $J$ Health Educ. 1999;30:S44-S51.

6. Roussos S, Fawcett S. A review of collaborative partnerships as a strategy for improving community health. Annu Rev Public Health. 2000;21:369-402.

7. Shediac-Rizkallah MC, Bone LR. Planning for the sustainability of community-based health programs: conceptual frameworks and future directions for research, practice and policy. Health Educ Res. 1998;13:87-108.

8. National 4-H Council. What is 4-H? https://4-h.org/about/what-is-4-h/. Accessed June 5, 2018.

9. National Institute of Food and Agriculture. 4-H Positive Youth Development. https://nifa.usda.gov/program/4-hpositive-youth-development. Accessed June 5, 2018.

10. National Institute of Food and Agriculture, US Department of Agriculture. Experiential Learning Model. https:// nifa.usda.gov/sites/default/files/ resource/Experiential-LearningModel.pdf. Accessed June 5, 2018

11. Bandura A. Social cognitive theory: an agentic perspective. Annu Rev Psychol. 2001;52:1-26.

12. Ostbye T, Zucker N, Krause S. Kids and adults now! Defeat obesity (KANDO): rationale, design and baseline characteristics. Contemp Clin Trials. 2011;32:461-469.

13. Barnes M. Solving the Problem of Childhood Obesity Within a Generation. Washington, DC: White House Task Force on Childhood Obesity Report to the President; 2010.

14. Skinner AC, Perrin EM, Skelton JA. Prevalence of obesity and severe obesity in US children, 1999-2014. Obesity (Silver Spring). 2016;24:1116-1123.

15. Hersch D, Perdue L, Ambroz T, Boucher J. The impact of cooking classes on food-related preferences, attitudes, and behaviors of school-aged children: a systematic review of the evidence. Prev Chronic Dis. 2014;11: E193.

16. Kochanska G, Kim S, Boldt L. (Positive) power to the child: the role of children's willing stance toward parents in developmental cascades from toddler age to early preadolescence. Dev Psychopathol. 2015;27(4 part 1):987-1005

17. Todd AS, Street SI, Ziviani J, Byrne NM, Hills AP. Overweight and obese adolescent girls: the importance of promoting sensible eating and activity behaviors from the start of the adolescent period. Int J Environ Res Public Health. 2015;12:2306-2329.

18. Fulkerson JA, Story M, Mellin A, Leffert N, Neumark-Sztainer D, French SA. Family dinner meal frequency and adolescent development: relationships with developmental assets and highrisk behaviors. $J$ Adolesc Health. 2006;39:337-345. 
19. Dinour L, Bergen D, Yeh M. The food insecurity-obesity paradox: a review of the literature and the role food stamps may play. J Am Diet Assoc. 2007;107: 1952-1961.

20. Townsend M, Peerson L, Love B, Achterberg C, Murphy S. Food insecurity is positively related overweight in women. J Nutr. 2001;131:17381745.

21. Franzen-Castle L, Colby SE, Kattelmann KK, et al. Development of the $i$ Cook 4-H curriculum for youth and adults: cooking, eating, and playing together for childhood obesity prevention. J Nutr Educ Behav. 2019;51: S60S68.

22. Reinehr T, de Souse G, Toschke A, Andler W. Long-term follow-up of cardiovascular disease risk factors in children after an obesity intervention. $A m \mathrm{~J}$ Clin Nutr. 2006;84:490-496.

23. Savoye M, Shaw M, Dziura J, et al. Effects of a weight management program on body composition and metabolic parameters in overweight children. JAMA. 2007;297:2697-2704.

24. McCallum Z, Wake M, Gerner B, et al. Outcome data from the LEAP (Live, Eat and Play) trial: a randomized controlled trial of a primary care intervention for childhood overweight/mild obesity. Int J Obes (Lond). 2007;31:630636.

25. Nemet D, Barkan S, Epstein Y, Friedland $\mathrm{O}$, Galit K, Eliakim A. Short- andlong-term beneficial effects of a combined dietary-behavioral-physical activity intervention for the treatment of childhood obesity. Pediatrics. 2005;115: 443-449.

26. Nitzke S, Kritsch K, Boeckner L, et al. A stage-tailored multi-modal intervention increases fruit and vegetable intakes of low-income young adults. Am J Health Promot. 2007;22:6-14.

27. Bates D, Maechler M, Bolker B, Walker S. Mixed-effects models using Ime4. J Stat Softw. 2015;67:1-48.

28. Random.org. True Random Number Service. http://RANDOM.org. Accessed January 19, 2019.

29. Kuczmarski RJ, Ogden CL, GrummerStrawn LM, et al. CDC growth charts: United States. Adv Data. 2000;314:127.

30. Hitze B, Bosy-Westphal A, Bielfeldt F, Settler U, Monig H, Muller MJ. Measurement of waist circumference at four different sites in children, adolescents, and young adults: concordance and correlation with nutritional status as well as cardiometabolic risk factors. Obes Facts. 2008;1:243-249.

31. Tanner J. Growth at Adolescence. 2nd ed. Oxford, UK: Blackwell Scientific; 1962.

32. Marshall WA, Tanner JM. Variations in pattern of pubertal changes in girls. Arch Dis Child. 1969;44:291-303.

33. Marshall WA, Tanner JM. Variations in the pattern of pubertal changes in boys. Arch Dis Child. 1970;45:13-23.

34. Department of Health and Human Services, National High Blood Pressure Education Program Working Group on High Blood Pressure in Children and Adolescents. A Pocket Guide to High Blood Pressure in Children and Adolescents. Bethesda, MD: National Institutes of Health; 2007.

35. National High Blood Pressure Education Program Working Group on High Blood Pressure in Children and Adolescents. The fourth report on the diagnosis, evaluation, and treatment of high blood pressure in children and adolescents. Pediatrics. 2004;114:555576.

36. Whelton P, Carey R, Aronow W, Casey D, Collins K. Guidelines for the prevention, detection evaluation and management of high blood pressure in adults: executive summary: a report of the American College of Cardiology/ American Health Association task force on clinical practice guidelines. $J \mathrm{Am}$ Coll Cardiol. 2017;17:735-1097.

37. Nutrition Quest. Block food screeners for ages 2-17. http://nutritionquest. com/assessment/list-of-questionnairesand-screeners/. Accessed June 5, 2018.

38. Cullen K, Watson K, Zakeri I. Relative reliability and validity of the Block Kids Questionnaire among youth aged 10 to 17 years. J Acad Nutr Diet. 2008;108:862-866.

39. Varni J, Seid M, Rode C. The Peds QL: measurement model for the pediatric quality of life inventory. Med Care. 1999;37:126-139.

40. National Cancer Institute. Quick Food Scan. 2000. https://epi.grants.cancer. gov/diet/screeners/fat/percent_energy. pdf?file $=/$ diet $/$ screeners $/$ fat $/$ percent_ energy.pdf. Accessed June 5, 2018.

41. National Institutes of Health. Eating at America's Table Study. https://epi. grants.cancer.gov/diet/screeners/fruitveg/allday.pdf?file=/diet/screeners/ fruitveg/allday.pdf. Accessed June 5, 2018.

42. Neumark-Sztainer D, Hannan P, Story M, Croll J, Perry C. Family meal patterns: associations with sociodemographic characteristics and improved dietary intake among adolescents. J Am Diet Assoc. 2003;103:317-322.

43. Fulkerson J, Neumark-Sztainer D, Story M. Adolescent and parent views of family meals. J Am Diet Assoc. 2006;106:526-532.

44. Kouneski EF. Family Assessment and the Circumplex Model. New Research Developments and Applications. Twin Cities, MN: University of Minnesota Press; 2000.

45. US Department of Health and Human Services Centers for Disease Control and Prevention. Health-related quality of life (HRQOL). http://www.cdc. gov/hrqol/data.htm. Accessed November 10, 2018.

46. Anliker J, Willis W, Mongomery S. The development and testing of the behavior checklist questions for the EFNEP Evaluation/Reporting System. https:// nifa.usda.gov/sites/default/files/ resource/Development $\% 20$ and $\% 20$ Testing $\% 20$ of $\% 20$ the $\% 20$ Behavior $\% 20$ Checklist\%20Questions.pdf. Accessed November 12, 2018.

47. Birch LL, Fisher JO, Grimm-Thomas K, Markey C, Sawyer R, Johnson S. Confirmatory factor analysis of the Child Feeding Questionnaire: a measure of parental attitudes, beliefs and practices about child feeding and obesity proneness. Appetite. 2001;36:201210.

48. Blumberg S, Bialostosky K, Hamilton William, Briefel R. The effectiveness of a short form of the household food security scale. Am J Public Health. 1999;89:1231-1234.

49. Sorenson AR, Kattelmann KK, Meendering JR, et al. Assessment of physical activity in 9- to 10-year-old children participating in a family-centered intervention. Topics Clin Nutr. 2015;30:159166.

50. US Department of Agriculture. Choose MyPlate. https://www.choosemyplate. gov/. Accessed June 13, 2018.

51. University of Nebraska-Lincoln. Fast Foods! https://marketplace.unl.edu/ ne $4 \mathrm{~h} /$ catalogsearch/result/?q=Fast +Foods+4H5000<http://4h.unl.edu/ web/4hcurriculum/4h5000. Accessed June 13, 2018. 
52. University of Nebraska-Lincoln. Youth in Motion. https://marketplace. unl.edu/ne4h/youth-in-motion.html. Accessed June 13, 2018.

53. National Institute of Food and Agriculture. 4-H youth development. https:// nifa.usda.gov/sites/default/files/ resource/4-H\%20Youth $\% 20$ Development.pdf. Accessed June 5, 2018.

54. Barton K, Wrieden W, Anderson A. Validity and reliability of a short questionnaire for assessing the impact of cooking skills intervention. J Hum Nutr Diet. 2011;24:588-595.

55. Baranowski T, Watson KB, Bachman C, et al. Self efficacy for fruit, vegetable and water intakes: expanded and abbreviated scales from item response modeling analyses. Int J Behav Nutr Phys Act. 2010;7:25.

56. Mathews DR. Development of a 3Pronged Approach to Evaluation for the iCook 4-H Project [dissertation]. Orono, ME: School of Food and Agriculture, University of Maine; 2015.

57. Mathews DR, Kunicki Z, Colby S, et al. Development and testing of program evaluation instruments for the iCook 4-H curriculum. J Nutr Educ Behav. 2019;51:S21-S29.

58. Dunn T, Baguley T, Brunsden V. From alpha to omega: a practical solution to the pervasive problem of internal consistency estimation. $\mathrm{Br} J$ Psychol. 2013;105:399-412.

59. Revelle W, Zinbarg RE. Coefficients alpha, beta, omega, and the glb: comments on Sijtsma. Psychometrika. 2009;74:145.

60. US Department of Agriculture. eXtension Online Campus. https://campus. extension.org/. Accessed January 21, 2019.

61. Century J, Rudnick M, Freeman C. A framework for measuring fidelity of implementation: a foundation for shared language and accumulation of knowledge. Am J Eval. 2010;31:199218.

62. Kuznetsova A, Brockhoff PB, Christensen RHB. ImerTest Package: tests in linear mixed effects models. J Stat Softw. 2017;82:1-26.

63. Gelman A, Hill J. Using Regression and Multilevel/Hierarchical Models. Cambridge, UK: Cambridge University Press; 2006.

64. Selvin S. Statistical Analysis of Epidemiologic Data. 3rd ed. New York, NY: Oxford University Press; 2004.
65. Hothorn T, Bretz F, Westfall P. Simultaneous inference in general parametric models. Biom J. 2008;50:346-363.

66. Cohen J. A power primer. Psychol Bull. 1992;112:155-159.

67. Cohen J. Statistical Power Analysis for the Behavioral Sciences. Hillsdale, NJ: Erlbaum; 1988.

68. Westfall J, Kenny DA, Judd CM. Statistical power and optimal design in experiments in which samples of participants respond to samples of stimuli. $J$ Exp Psychol Gen. 2014;143:2020-2045.

69. Van Buuren S, Groothuis-Oudshoorn K. Mice: multivariate imputation by chained equations in R. J Stat Softw. 2011;45:1-67.

70. Singer JD, Willett JB. Applied Longitudinal Data Analysis: Modeling Change and Event Occurence. Oxford UK: Oxford University Press; 2003.

71. Müller S, Scealy JL, Welsh AH. Model selection in linear mixed models. Stat Sci. 2013;28:135-167.

72. Olfert M, Famodu O, Flanagan S, et al. The effect of $i$ Cook 4-H, a childhood obesity prevention program, on blood pressure and quality of life in youth and adults: a randomized control trial. $J$ Child Obes. 2018;3:1-8.

73. Barr-Anderson DJ, Singleton C, Cotwright CJ, Floyd MF, Affuso O. Outside-of-school time obesity prevention and treatment interventions in African American youth. Obes Rev. 2014;15 (suppl 4):26-45.

74. Rinderknecht K, Smith C. Social Cognitive Theory in an after-school nutrition intervention for urban Native American youth. J Nutr Educ Behav. 2004;36:298-304.

75. Davis JN, Ventura EE, Cook LT, Gyllenhammer LE, Gatto NM. LA Sprouts: a gardening, nutrition, and cooking intervention for Latino youth improves diet and reduces obesity. $J$ Am Diet Assoc. 2011;111:1224-1230.

76. Annesi JJ, Walsh SM, Greenwood BL, Mareno N, Unruh-Rewkowski JL. Effects of the youth Fit 4 Life physical activity/nutrition protocol on body mass index, fitness and targeted social cognitive theory variables in 9- to 12-year-olds during after school care. J Paediatr Child Health. 2017;53:365-373.

77. Louie JCY, Flood WM, Hector DJ, Rangan AM, Gill TP. Dairy consumption and overweight and obesity: a systematic review of prospective cohort studies. Obes Rev. 2011;12:e582-e592.
78. Keast DR, Hill Gallant KM, Alberson AM, Gugger CK, Holschuh NM. Associations between yogurt, dairy, calcium, and vitamin D intake and obesity among US children aged 8-18 years: NHANES, 2005-2008. Nutrients. 2015;7:1577-1593.

79. Gatto N, Ventura E, Cook L, Gyllenhammer L, Davis J. LA Sprouts: a garden-based nutrition intervention pilot program influences motivation and preferences for fruits and vegetables in Latino youth. $J$ Acad Nutr Diet. 2012;112:913-920.

80. Slusser W, Sharif M, Erausquin J, Kinsler J, Collin D, Prelip M. Improving overweight among at-risk minority youth: results of a pilot intervention in after-school programs. J Health Care Poor Underserved. 2013;24(2 suppl):1224.

81. Gortmaker SL, Lee RM, Mozaffarian RS, et al. Effect of an after-school intervention on increases in children's physical activity. Med Sci Sports Exerc. 2012;44:450-457.

82. Robson S, Stough C, Stark L. The impact of a pilot cooking intervention for parent-child dyads on the consumption of foods prepared away from home. Appetite. 2016;99:177-184.

83. Olfert MD, King SJ, Hagedorn RL, et al. Ripple effects mapping outcomes of a childhood obesity prevention program for youth and adult dyads using a qualitative approach. iCook 4-H. J Nutr Educ Behav. 2019;51:S41S51.

84. Emery M, Higgins L, Chazdon S, Hansen D. Using ripple effect mapping to evaluate program impact: choosing or combining the methods that work best for you. $J$ Ext. 2015;53:2TOT1.

85. Faith MS, Berkowitz RI, Stalling VA, Kerns J, Storey M, Stunkard AJ. Parental feeding attitudes and styles and child body mass index: prospective analysis of a gene-environment interaction. Pediatrics. 2004;114:e429-e436.

86. Hennessy E, Hughes SO, Goldberg JP, Hyatt RR. Parent behavior and child weight status among a diverse group of underserved rural families. Appetite. 2010;54:369-377.

87. McElrone M, Colby SE, Franzen-Castle L, Olfert M, Kattelmann K, White A. Prevalence and predictors of household food insecurity among adult/ youth dyads at the initiation of the $i$ Cook 4-H two-year obesity prevention 
study. J Child Obes. 2018;3(suppl 1): $1-11$.

88. Chester A, Dooley D. West Virginia University's health sciences and technology. J Higher Educ Outreach Engagem. 2011;15:87-99.

89. Hagedorn RL, White JA, FranzenCastle L, et al. Teens implementing a childhood obesity prevention program in the community: feasibility and perceptions of a partnership with HSTA and iCook 4-H. Int J Environ Res Public Health. 2018;15:E934.

90. Kattelmann KK, Meendering JR, Hofer EJ, et al. The iCook 4-H Study: report on physical activity and sedentary time in youth participating in a multicomponent program promoting family cooking, eating, and playing together. J Nutr Educ Behav. 2019;51:S30-S40.

91. Olfert MD, Hagedorn RL, Barr ML, et al. Dissemination using infographic reports depicting program impact of a community-based research program: eB4CAST in iCook 4-H. J Nutr Educ Behav. 2019;51: S52-S59. 